\title{
The spindle matrix revisited
}

\begin{abstract}
DOI:
10.1038/nrm1927

\section{URLs}

Lamin-B

http://ca.expasy.org/uniprot/

P10999

NuMA

http://ca.expasy.org/uniprot/

P70012

XMAP215

http://ca.expasy.org/uniprot/ Q9PT63

TPX2

http://ca.expasy.org/uniprot/ Q6NUF4

Chromosome segregation during mitosis is coordinated by the mitotic spindle, which requires the joint activities of microtubules, microtubule-binding proteins and chromosomes. Although it has been suggested that a structural scaffold the so-called spindle matrix - tethers spindle-assembly factors (SAFs) and supports spindle assembly, the nature of this scaffold has remained elusive. Now, Yixian Zheng and colleagues report in Science that the intermediate-filament protein lamin-B is required for spindle assembly, and they propose a structural role for lamin-B in the spindle matrix.

Lamins have regulatory and structural functions in interphase nuclei, but previous studies indicated that a fraction of lamin-B might also
\end{abstract}

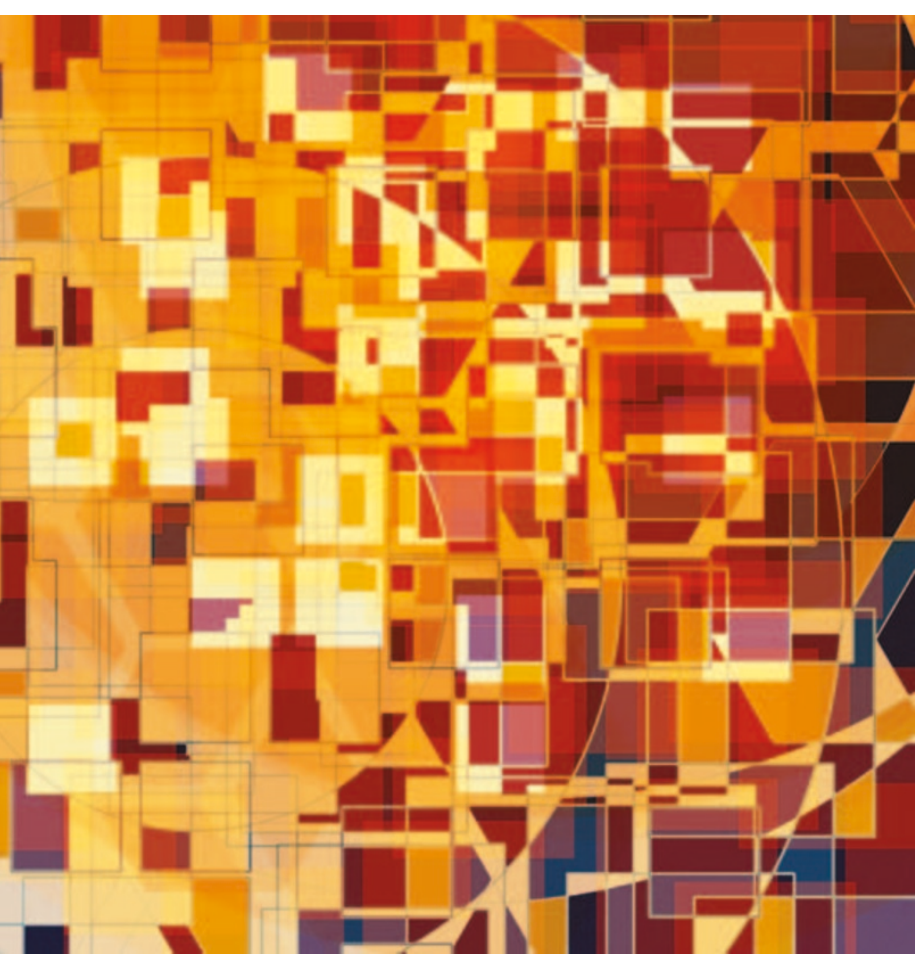

associate with the mitotic spindle and therefore might have a role in mitosis. Zheng and colleagues showed, using immunofluorescence, that lamin-B associates with mitotic spindles in Xenopus laevis egg extracts and $\mathrm{HeLa}$ cells. Depletion of lamin-B by RNA interference in HeLa cells led to an increase in defects that are associated with spindle assembly and function, which indicates that lamin-B might function in spindle assembly. To rule out the possibility that the spindle defects were an indirect result of the perturbation of interphase functions, the authors carried out experiments in M-phase X. laevis egg extracts, and obtained similar results.

Next, the authors showed that lamin-B assembled into a matrix-like network during mitosis, and that this process depended on the presence of the GTP-bound form of the small GTPase Ran. Spindles were assembled in $X$. laevis egg extracts in the presence or absence of RanGTP, and the spindle microtubules were subsequently depolymerized with nocodazole. This revealed a lamin-Bspecific fibrillar-granular matrix, which formed only in the presence of RanGTP. A possible explanation for the requirement of RanGTP is that the nuclear-import proteins importin- $\alpha$ and $-\beta$ interfere with the assembly of the lamin- $B$ matrix, and that RanGTP displaces importin- $\alpha$ and $-\beta$ from proteins such as lamin-B that are required for matrix assembly.

Double-immunofluorescence analyses of the lamin-B matrix showed the presence of both lamin-B and SAFs (poly(ADP-ribose), NuMA, Eg5, XMAP215 and TPX2) in the same matrix. Although the depletion of lamin-B inhibited the formation of matrix structures that contained SAFs, the depletion of either Eg5 or XMAP215 still allowed the formation of lamin-B matrices that contained other SAFs. These findings indicate that lamin-B is required for the assembly of a spindle-associated matrix.

Zheng and co-workers were able to isolate the lamin-B matrix from M-phase egg extracts that were treated with nocodazole, and could indeed detect the presence of lamin-B and several SAFs. When incubated with pure tubulin, these isolated matrices caused the nucleation of microtubules, which tethered to the matrices. By contrast, no microtubules assembled in the absence of the matrices. Importantly, the matrices from which XMAP215 was absent were unable to promote microtubule assembly. This implies that the lamin-B matrices can promote spindle assembly by tethering SAFs.

Finally, the authors showed that lamin-B mutants that were unable to form proper interphase nuclear lamina disrupted spindle assembly. Also, the localization of SAFs on the microtubule structures in the matrices that contained mutant lamin-B was abnormal. Therefore, the proper assembly of the lamin-B matrix is essential for the organization and localization of SAFs on spindles during mitosis.

This work indicates that lamins might have nuclear structural roles not only in interphase, but also in mitosis, and sheds important new light on the elusive spindle matrix.

\section{Arianne Heinrichs}

ORIGINAL RESEARCH PAPER Tsai, M.-Y. et al. A mitotic lamin B matrix induced by RanGTP required for spindle assembly. Science 16 March 2006 (doi:10.1126/science.1122771) 